\title{
Scaling Study of Cavitation Pitting from Cavitating Jets and Ultrasonic Horns
}

\author{
Arvind Jayaprakash ${ }^{1}$, Jin-Keun Choi ${ }^{1}$, Georges L. Chahine ${ }^{1}$, \\ Farrel Martin ${ }^{2}$, Martin Donnelly ${ }^{3}$, Jean-Pierre FRANC ${ }^{4}$, and Ayat Karimi ${ }^{5}$
}

\begin{abstract}
Cavitation erosion prediction and characterization of cavitation field strength are of interest to industries suffering from cavitation erosion detrimental effects. One means to evaluate cavitation fields and materials is to examine pitting rates during the incubation period, where the test sample undergoes localized permanent deformations shaped as individual pits. In this study, samples from three metallic materials, an Aluminum alloy (Al7075), a Nickel Aluminum Bronze (NAB) and a Duplex Stainless Steel (SS A2205) were subjected to a vast range of cavitation intensities generated by cavitating jets at different driving pressures and by an ultrasonic horn. The resulting pitted sample surfaces were examined and characterized with a noncontact 3D optical scanner and the resulting damage computer-analyzed. A statistical analysis of the pit population and its characteristics was then carried out. It was found that the various cavitation field strengths can be correlated to the measured pit distributions and that two characteristic quantities: a characteristic number of pits per unit surface area and unit time, and a characteristic pit diameter or a characteristic pit depth can be attributed to a given "cavitation intensity level". This characterization concept can be used in the future to study the cavitation intensity of the full scale and to develop methods of full scale predictions based on model scale erosion data.
\end{abstract}

Keywords: Cavitation erosion; Erosion testing; Steel; Non-ferrous metals; Erosion modeling

\footnotetext{
1 DYNAFLOW, INC., Jessup, Maryland, U.S.A. email: jkchoi@dynaflow-inc.com

2 Naval Research Laboratory, email: farrel.martin@nrl.navy.mil

3 Naval Surface Warfare Center Carderock Division, martin.donnelly@navy.mil

4 Grenoble University (LEGI) Grenoble, France, email: Jean-Pierre.Franc@legi.grenoble-inp.fr

5 EPFL, Lausanne, Switzerland, email: ayat.karimi@epfl.ch
} 


\section{Introduction}

Evaluation of a new material's resistance to cavitation erosion often relies on comparative laboratory studies involving accelerated erosion tests. This is the case because scaled erosion tests are either not possible, too slow, or because cavitation erosion scaling is still not fully understood scientifically. Accelerated erosion tests to evaluate a material or select one material among several, by definition, involves subjecting material samples to a cavitation field that produces measurable erosion over a short period of time. This, almost by definition, involves a different type of cavitation erosion than what is present in the actual cavitation field the tested material is destined to be subjected to. Casually, the laboratory determined ranking of the materials in the accelerated erosion tests is practically assumed to hold in the real application field. However, evidence exists that this may not always be the case, since some materials at least react differently to cavitation fields of different strength [1-4].

Fundamentally, the mechanical process of cavitation erosion results from successive individual and collective cavity collapses, which generate local high amplitude, short duration loads. The overall picture is that bubble nuclei in the liquid grow explosively in low pressure regions forming cavitation bubble clouds [5-9]. These subsequently collapse generating very high local pressures and temperatures [10-11]. In addition, when the bubbles collapse very close or at the material surface, micro reentrant jets from bubble large deformations vector towards the material and impact its surface [12-15]. When the pressure loads exceed the elastic limit of the material, the material undergoes permanent deformations leaving microscopic pits [16]. This initial incubation period of the material response to the erosion cavitation flow field does not involve any mass loss. With repeated impacts, hardening of the material surface layer develops, the deformation of the material accumulates, and finally microfailures occur resulting in material removal and thus weight loss.

One method to investigate a portion of the above dynamics is to conduct pitting tests, i.e. short duration tests during the incubation period, where isolated (not overlapping) pits can be identified and characterized [1]. By doing so, the material is used as a recorder of the highest pressures in the cavitating field, since each material acts as a high pass filter and records as pits only the cavitating field pressure peaks at the material surface that exceed its yield stress and plastically deform permanently. Observation of pits for the purpose of evaluating the cavitation field intensity dates back to the early 1900's when Parsons and Cook [17] observed the depth and dimensions of the pitted areas, and researchers reported the pitting location relative to the cavitation cloud shape and statistics such as the number and the depth of pitting [18-20]. Knapp [19-21] introduced the idea that the pits could be used to understand the intensity of the cavitation field. Similar ideas of observing pits to represent the cavitation field was used in hydraulic turbine cavitation erosion studies [22], where it was found that the cavitation aggressiveness in the full scale is more severe than that of the model scale. Recent studies using pitting tests includes the use of thin copper foil in order to capture relative small magnitude impacts [23], analysis of pitting to determine the impact loads for a modeling effort for ductile metals [24], evaluation of flow aggressiveness in the study of hydraulic turbine flows [25], and applications to the erosion studies of marine propellers $[26,27]$. With the advance of modern imaging and micro-measurement technologies, recent studies reveal more details of the pit shapes and statistics [28,29]. However, these studies did not provide a unified description of the cavitation field based on the pitting tests.

In this paper, we model the pit statistics as an attempt to identify parameters that characterize the aggressiveness of a cavitation field. Three different materials, an Aluminum alloy, a Nickel Aluminum Bronze, and a Duplex Stainless Steel were subjected to vastly different intensities of cavitation field generated by acoustic horn [30] and by DYNAJETS ${ }^{\circ}$ cavitating jets $[4,31,32]$ at different driving pressures. These were provided by the Naval Surface Warfare Center, Carderock Division. The pit population and sizes resulting from the tests in these different cavitation fields were analyzed and statistically processed. Based on this analysis, we propose a model that describes the pitting statistics with a small number of parameters, which in the future can be used to define the cavitation field intensity level. The above three materials are the same three materials used in Franc et al. [33], and results in this paper can be used as a comparative study with [33] of various cavitation erosion generation methods. The study described in this paper is an effort to deduce a relationship between the cavitation field intensity and the pitting statistics. This is a part of an on-going rather large effort, in which we are investigating experimentally and modeling numerically the erosion process in order to relate 
the material erosion to the cavitation field intensity in a predictive manner.

\section{Experimental Setup and Procedure}

In order to study cavitation erosion in a controlled environment and in an accelerated manner, several laboratory techniques to generate cavitation have been used in the past. These techniques include the utilization of ultrasonic vibration to generate the cavitation, cavitation flow loops with strong separating flows, rotating disks, venturi cavitating flows, vortex generators, and submerged cavitating jets. Some of these techniques were standardized and resulted in American Society for Testing and Materials (ASTM) Standards [30] such as G-32 "Test Method for Cavitation Erosion Using Vibratory Apparatus" and G-134 "Test Method for Erosion of Solid Materials by a Cavitating Liquid Jet". In this study, the ultrasonic test method following G32 and cavitating jet tests were conducted at DYNAFLow to investigate the incubation period. The time evolution of the weight loss of the three considered materials was documented in [32].

\subsection{Ultrasonic Cavitation Erosion Testing}

We followed in our test the prescribed ultrasonic cavitation tests ASTM G-32 method [30]. The cavitation is generated by a vibratory device employing a magnetostrictive ultrasonic horn. The acoustic horn was operated at $20 \mathrm{kHz}$ with a peakto-peak amplitude of $50 \mu \mathrm{m}$. The amplitude was set using a bifilar microscope and maintained at that value throughout testing. The samples were held in place with fixed sample holders inside a 2,000 $\mathrm{ml}$ beaker filled with distilled water and with the horn tip submerged $12 \mathrm{~mm}$ beneath the free surface. The beakers were immersed in a water bath maintained at $25 \pm 2{ }^{\circ} \mathrm{C}$. In the "alternative" G32 test configuration (also known as a stationary specimen method), the horn tip is placed at a small distance from the stationary material sample, here at $0.5 \mathrm{~mm}$ below the tip of the horn, and the cavitation cloud was generated in between the two. We used $25.4 \mathrm{~mm} \times 25.4 \mathrm{~mm}$ square samples of the material to be tested.

The temperature, liquid beaker volume, horn tip submergence beneath the free surface, frequency, and amplitude of the oscillations are all prescribed by the ASTM G-32 method. In the direct G32 method the cavitation cloud collapses in a hemispherical way toward the material, while in the modified method, the cavitation bubble cloud collapses in a cylindrical way. It is well known from previous studies [34] that the cloud cavitation collapsing cylindrically is much less erosive than the hemispherically collapsing cavitation clouds, thus the alternative methods produce less erosive results than the direct method.

Usually, for mass loss tests, the procedure is to expose the sample to cavitation for a given period of time, stop the test, remove the sample, and record weight to enable measurement of weight loss as a function of time. The sample is then returned for additional testing. In the pitting tests, only one interval of above mass loss test was conducted. The samples were polished up to a mirror like surface so that the surface scan conducted later will have less noise from the existing roughness of the surface. The facility at Naval Research Laboratory (NRL) was used to polish the samples. The specimens were prepared using SiC metallographic papers, starting with 240 grit and increasing fineness of 400,600, 1200 and 1500 , followed by diamond slurry polishes of decreasing grit diameter of 10, 3, 1 and .01 microns. The final step in polishing was performed on a vibratory polisher for a period of 12 hours. The polished samples were kept individually in a container, and the surface was not touched by any other objects until the beginning of the test. As soon as the test is completed, the sample was dried completely by air blower and then stored individually in a container. The tested surface was not touched until it was examined by the optical scanner.

\subsection{DYNAJETS ${ }^{\oplus}$ Cavitating Jet Tests}

The cavitating jet erosion tests used DYNAJETS ${ }^{\circledR}$ cavitating jet nozzles, a sample holder, a water tank, and a pump. The sample holder ensures that the sample is held in place during the pitting tests (Figure 1). The pitting test procedure using this jet cavitation was as follows:

(a) The sample surface was polished up to mirror like surface.

(b) The sample was exposed to the cavitating jet for a predetermined period of time.

(c) The sample was taken out from its holder, and carefully dried.

(d) The surface was scanned using an optical profilometer.

The sample preparation and post-test procedures was the same to that of the ultrasonic tests as described in the preceding section. 


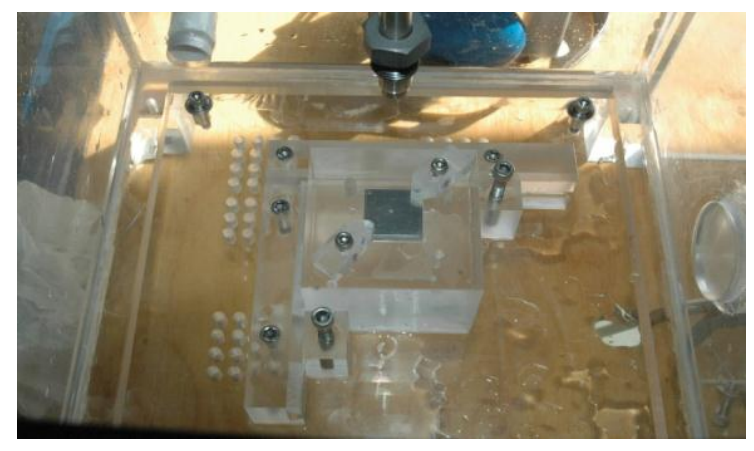

Figure 1. Nozzle and sample holder in DYNAFLOW's "7 ksi - 5 gpm" cavitation jet erosion test loop.

\section{Materials Tested}

Three materials were selected for tests under this program: Aluminum alloy 7075 - T651, Nickel Aluminum Bronze Alloy (NAB), and Duplex Stainless Steel SS A2205. The main properties of the tested materials are shown in Table 1.

We also conducted Split Hopkinson Pressure Bar (SHPB) tests on these materials to measure the high strain rate properties of these materials. Figure 2 compares the engineering stress vs. engineering strain curves for Aluminum alloy 7075 at different strain rates $(1200 / s$ $1825 / \mathrm{s}$ ) and a literature data for a near zero strain rate [35]. The SHPB curves show some oscillations, which are typical especially near the end of curves. This portion of data were obtained when the sample was about to detach from the bar.

\begin{tabular}{|c|c|c|c|}
\hline Material & $\begin{array}{c}\text { Aluminum } \\
\text { Alloy } \\
7075 \\
\mathrm{~T} 651(\mathrm{Al})\end{array}$ & $\begin{array}{c}\text { Nickel } \\
\text { Aluminum } \\
\text { Bronze } \\
\text { (NAB) }\end{array}$ & $\begin{array}{c}\text { Duplex } \\
\text { Stainless } \\
\text { Steel } \\
2205 \\
(\mathrm{SS})\end{array}$ \\
\hline $\begin{array}{c}\text { Tensile } \\
\text { Yield } \\
\text { Strength }\end{array}$ & $503 \mathrm{MPa}$ & $355 \mathrm{MPa}$ & $510 \mathrm{MPa}$ \\
\hline $\begin{array}{c}\text { Ultimate } \\
\text { Tensile } \\
\text { Strength }\end{array}$ & $572 \mathrm{MPa}$ & $683 \mathrm{MPa}$ & $750 \mathrm{MPa}$ \\
\hline $\begin{array}{c}\text { Modulus of } \\
\text { Elasticity }\end{array}$ & $71.7 \mathrm{GPa}$ & $115 \mathrm{GPa}$ & $200 \mathrm{GPa}$ \\
\hline $\begin{array}{c}\text { Elongation } \\
\text { at Break }\end{array}$ & $11 \%$ & $10 \sim 18 \%$ & $35 \%$ \\
\hline
\end{tabular}

Table 1. Tested materials and main properties.

The Aluminum alloy 7075 showed slightly larger strain at high strain rates than the strain at the corresponding static loading. Near zero strain, the average slope of these curves are obtained and compared with the literature values of the static modulus of elasticity in Table 2 . The moduli of elasticity deduced from the slope of the stressstrain curves from the SHPB were comparable to the static values from the literature and from nano-indentor measurements conducted at EPFL [36,37].

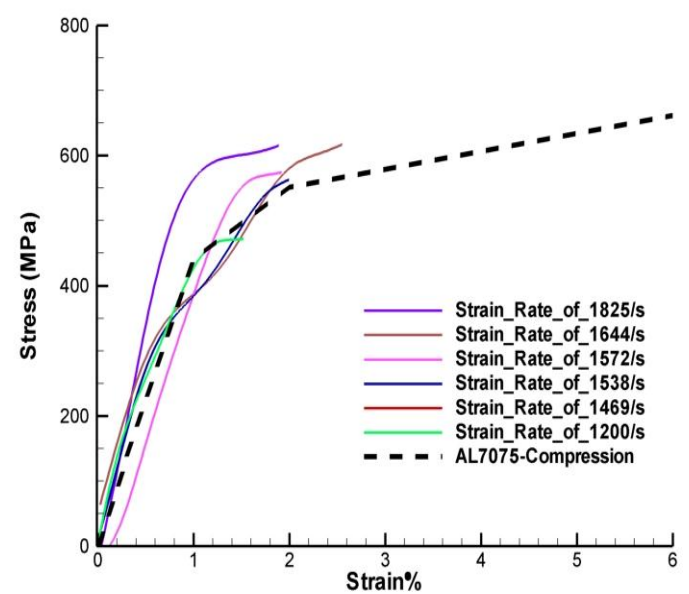

Figure 2. Comparison of stress-strain curves of Al 7075 obtained from Split Hopkinson Pressure Bar (SHPB) tests and a reported curve for a near zero strain rate from literature [35].

\begin{tabular}{|c|c|c|c|}
\hline Source & $\begin{array}{c}\text { Aluminum } \\
\text { Alloy } \\
7075 \\
\text { T651 }\end{array}$ & $\begin{array}{c}\text { Nickel } \\
\text { Aluminum } \\
\text { Bronze } \\
\text { Alloy } \\
\text { (NAB) }\end{array}$ & $\begin{array}{c}\text { Duplex } \\
\text { Stainless } \\
\text { Steel } \\
2205 \\
\text { (SS) }\end{array}$ \\
\hline $\begin{array}{c}\text { Literature } \\
\text { [37] }\end{array}$ & $71.7 \mathrm{GPa}$ & $120 \mathrm{GPa}$ & $200 \mathrm{GPa}$ \\
\hline $\begin{array}{c}\text { DYNAFLOW } \\
\text { SHPB } \\
\text { tests }\end{array}$ & $67.6 \mathrm{GPa}$ & $127 \mathrm{GPa}$ & $190 \mathrm{GPa}$ \\
\hline $\begin{array}{c}\text { Nano- } \\
\text { indentor } \\
\text { tests } \\
\text { (EPFL) } \\
{[36]}\end{array}$ & $86.0 \mathrm{GPa}$ & $150 \mathrm{GPa}$ & $215 \mathrm{GPa}$ \\
\hline
\end{tabular}

Table 2: Comparison of the modulus of elasticity for the three materials tested.

\section{$4 \quad$ Pit Analysis Techniques}

\subsection{Procedures}

The $25.4 \mathrm{~mm} \times 25.4 \mathrm{~mm} \times 6.35 \mathrm{~mm}$ mirrorpolished material samples were subjected to 
cavitation fields of different intensities for predetermined time durations. The central jet impact area where maximum pitting occurred was chosen for detailed analysis. Optical scanning profilometry provided by the Naval Research Laboratory was used to measure very carefully the surface of the pitted sample. The optical scans were conducted over an area of $1 \mathrm{~mm} \times 1 \mathrm{~mm}$. The used optical scanner Alicona InfiniteFocus G4 with 100x optical objective lens, was capable of producing very high resolution data [38]. The interrogation mesh size was selected to be $1.5 \mu \mathrm{m} \times 1.5 \mu \mathrm{m}$. Figure 3 shows a contour plot of the surface data obtained from such an optical scan of the pitted surface.

Despite the fact that the surface was highly polished, surface level fluctuations can be seen in the surface as a result of polishing and machining marks, and could interfere with automatic measurement if not filtered. This required us to impose a threshold as a cut-off depth, $d_{t h}$, that was applied during post-process of the measured data; i.e. all elevations higher than $d_{t h}$, measured from the averaged zero level, were ignored or cut-off. One has however to realize that this threshold affects the statistical results as discussed later. In addition to the above, a few very deep pits noticed in the optical scan (as in Figure 4) were attributed to known artifacts caused by the optical measurement technique, and these erroneous data were filtered out.

\subsection{Pit Counting}

The surface scanning profilometer generates a full 3D discretized description of the eroded material surface, $z(x, y)$. From this discretized mathematical description, we derived using various geometry or particle analysis software statistical analyses of various quantities of interest here: number of pits, areas, volumes, equivalent diameter, average depth, shape factor, etc. of the pits. Because of the selection of advancing stages of very early pitting, very little pitting overlap occurred and individual pits could be easily identified, and the geometric characteristics of each individual pit could be accurately measured and used in the statistical analysis.

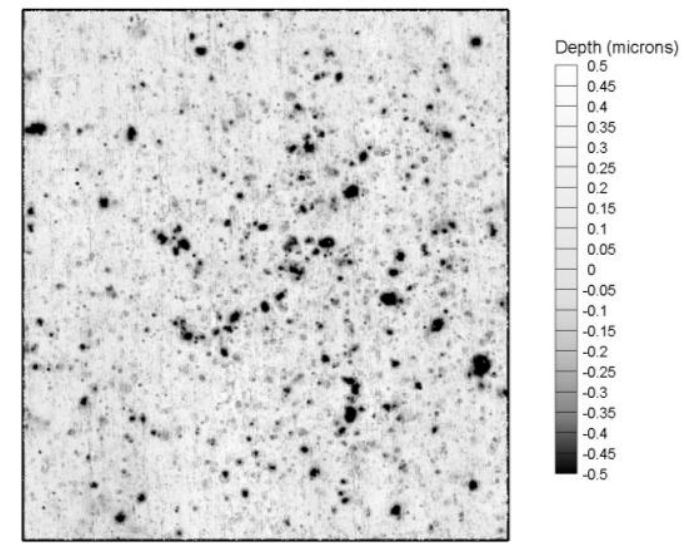

Figure 3. Typical data obtained from optical scanning of a pitted sample. Shown here is the actual $1.587 \mathrm{~mm} \times 1.719 \mathrm{~mm}$ scan of Al 7075 sample pitted under a 1,100 psi DYNAJETS ${ }^{\circledR}$ for a duration of $2 \mathrm{~min}$.

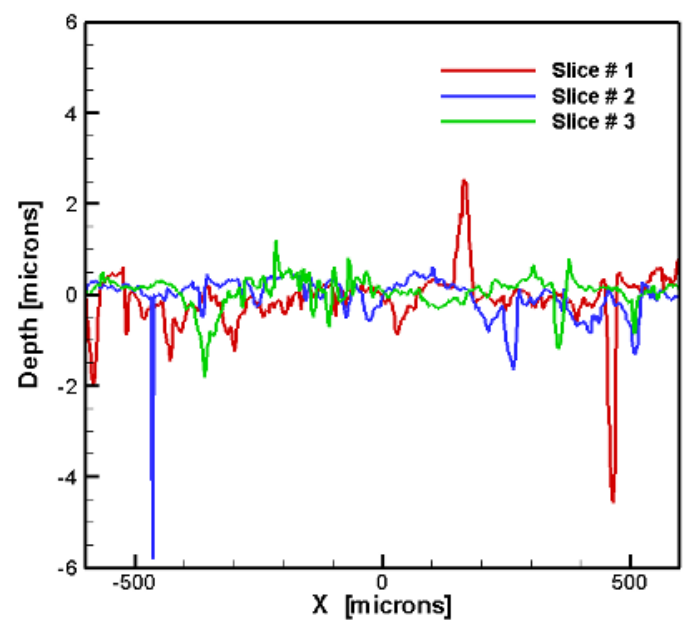

Figure 4. Typical surface levels in a vertical cut plane obtained from optical scan of pitted samples. This profile is obtained from the surface shown in Figure 3.

\begin{tabular}{|c|c|c|}
\hline $\begin{array}{c}\text { Data } \\
\text { Name }\end{array}$ & Computed Data & Unit \\
\hline $\begin{array}{c}\text { Pitting } \\
\text { Rate }\end{array}$ & $N=\frac{\text { number of pits }}{\text { exposure time } \times \text { analyzed surface }}$ & $\begin{array}{c}\text { Pits/ } \\
\mathrm{mm}^{2} / \\
\mathrm{S}\end{array}$ \\
\hline $\begin{array}{c}\text { Deform } \\
\text { ation } \\
\text { Depth } \\
\text { Rate }\end{array}$ & $\dot{h}=\frac{\text { volume of pits }}{\text { exposure time } \times \text { analyzed surface }} \mu \mathrm{m} / \mathrm{h}$ \\
\hline
\end{tabular}

Table 3: Measured and computed data.

\subsection{Cumulative Pitting Rate versus Pit Diameter}

From the scanned profilometer measurements, an average pitting rate can be defined as the cumulative number of pits after a given exposure time, $t_{\text {exp }}$, divided by $t_{\text {exp }}$. The 
actual instantaneous rate is harder to measure and would require many time measurement points. The deformation depth rate is also defined similarly from the measured average pit depth, defined as the ratio of pit volume to pit area (Table 3).

The counted pits can be classified according to their diameter. The cumulative diameter distribution function of the pitting rate can be defined as $N(D)$, where $N$ is the number of pits per unit area per unit time counted with a diameter larger than the value $D$. We have found that the cumulative distribution of pitting rate can be fitted reasonably well by a Weibull distribution, expressed by the following mathematical function:

$$
N=N^{*} \mathrm{e}^{-\left(D / D^{*}\right)^{k}},
$$

where $N^{*}$ is a characteristic number of pits, $D^{*}$ a characteristic diameter of the pits, and $k$ is a shape parameter. These three parameters are determined to best fit the measured data. In this study we defined the best fit using the least square method, minimizing the residual, $R$, of the fit defined by:

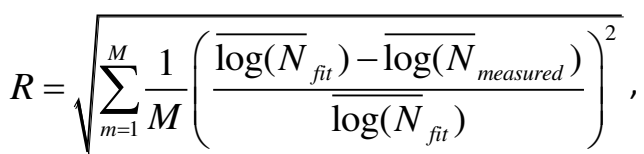

where $N_{\text {fit }}$ is the curve fit value, and $N_{\text {measured }}$ is the actually measured data.

\section{Effect of Cutoff Depth on Pitting Rates}

As mentioned earlier, in order to remove the noise from the measured surface geometry, a cut-off depth was applied in the scanned profilometer data post-processing, and this procedure affects by definition the characteristics of the detected pits. Hence, it is important to study the influence of the threshold value on the pit statistics.

As an example of the influence of the cutoff depth, Figure 5 shows the identified pits for each choice of the threshold. In the images, the identified pits are marked in red, and one can see that when the threshold value is lowered more pits are identified and individual pit areas increase, which is obvious from geometrical considerations: larger threshold values filter all shallow pits, and detect a smaller value of the surface area of the pits since the shape of these can be idealized as bowls or inverted cones. As a result, the pit density increases when the cut-off depth decreases.
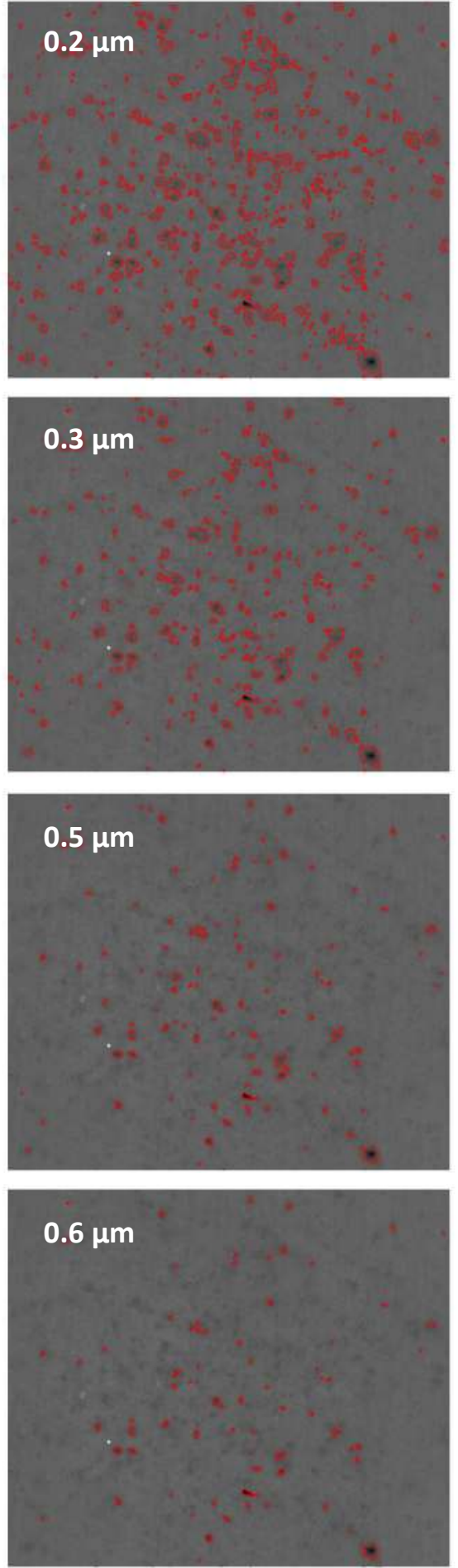

Figure 5. Influence of the cut-off depths $(0.2,0.3$, 0.5 , and $0.6 \mu \mathrm{m}$ ) on the identified pits. The identified pits are marked in red and the gray level is an indicator of the local depth.

Figure 6 compares the cumulative number of pits versus pit diameter obtained with six different depth threshold values. It can be observed from the figure that choosing a lower cut-off depth resulted in higher pitting rate 
probably due to the detection of surface roughness as erroneous pits. And on the other hand, more and more shallow pits were discarded with increasing cut-off, resulting in loss of smallest pits for further statistic analysis. Hence, it is important to select the cut-off depth threshold such that a statistically significant number of real, nonoverlapping pits can be identified. By comparing the raw image of the pitted surface and the detected pits at different cut-off depth, we concluded that $0.3 \mu \mathrm{m}$ cut-off is a reasonable choice that captures most of visually discernable pits. The $0.3 \mu \mathrm{m}$ cut-off is also above the noise of the virgin surface left after the final polish by the $0.01 \mu \mathrm{m}$ diamond slurry. We present below results for $0.3 \mu \mathrm{m}$ and $0.5 \mu \mathrm{m}$.

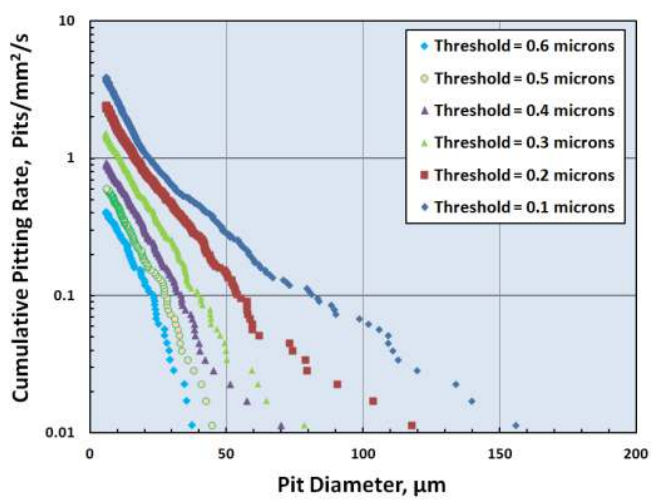

Figure 6. Influence of depth cut-off threshold on the pitting rate. Data shown is for SS A2205 pitted with 4000 psi cavitation jet for 1 minute.

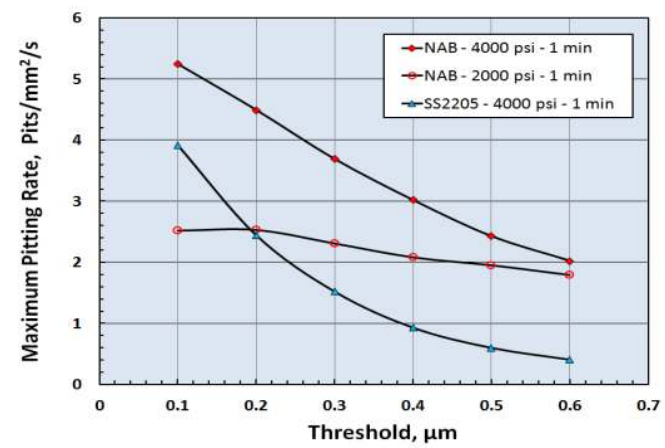

Figure 7. Effect of various threshold levels on pitting rate analysis for the three materials studied.

Figure 7 shows the maximum pitting rates (i.e. pitting rates for $D \geq 0.5 \mu \mathrm{m}$ ) for selected tested materials and pressures as functions of the cutoff depth value. The trend of detecting more number of pits as the threshold decreases is common for the two NAB samples and the SS sample. In this study, based on the results obtained, a cut-off depth of $0.3 \mu \mathrm{m}$ selected as a good compromise. Additionally, data for cut-off depth of $0.5 \mu \mathrm{m}$ is also presented for comparison purposes.

\section{Effect of Cavitation Intensity (Jet Pressure) on Pitting Rate}

We present here results of tests carried out at different jet pressures varying from 700 psi to 7000 psi (or jet speeds from about $250 \mathrm{ft} / \mathrm{s}$ to 850 $\mathrm{ft} / \mathrm{s})$. As discussed earlier, the pitting test results can be expressed by Weibull distributions with a shape parameter, $k$, a characteristic diameter, $D *$ and a characteristic pitting rate, $N *$. The effect of the jet pressure (or the cavitation intensity) on these parameters and, as a consequence, on the whole distribution function of pitting rate versus pit diameter is investigated in this section.

Figure 8 and Figure 9 show the pit distributions at different jet speeds for stainless steel A2205 samples respectively for cutoff depths in the analysis of the pits of $0.3 \mu \mathrm{m}$ and $0.5 \mu \mathrm{m}$. In all cases the jets were discharged at atmospheric pressure. For all case, the Weibull fits are acceptable and cover the full range of pit sizes including the larger diameter values, which correspond to rare high intensity events. The shape parameter, $k$, found to fit the data best was $k=0.74$ for the cutoff depth of $0.3 \mu \mathrm{m}$ and $k=0.85$ for the cutoff depth of $0.5 \mu \mathrm{m}$.

Figure 10 and Figure 11 show that curve fits with $k=1.0$, as used in [33], fit well the data for the smaller diameters but deviate for the more intense event which produce the larger diameter pits.

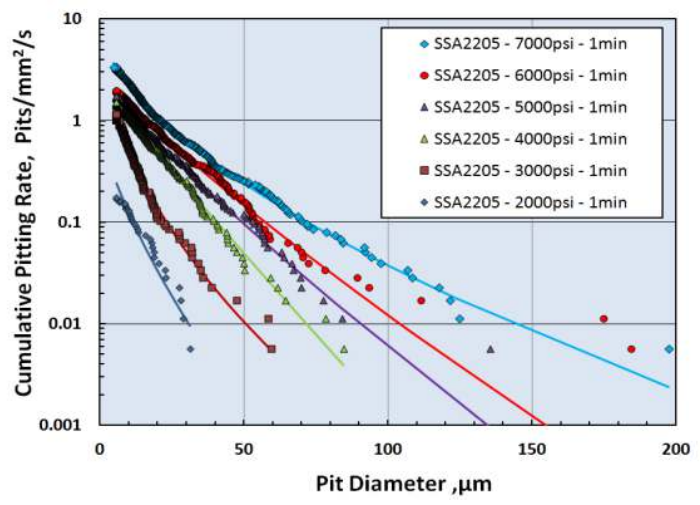

Figure 8: Cumulative pitting rate as a function of pit diameter for different values of the jet pressure on duplex stainless steel A2205 (cut-off depth: $0.3 \mu \mathrm{m})$. Curve fits correspond to the threeparameter Weibull distributions,

$$
N=N^{*} \exp \left[-\left(D / D^{*}\right)^{k}\right] \text {, with all three }
$$

parameters fitted. 


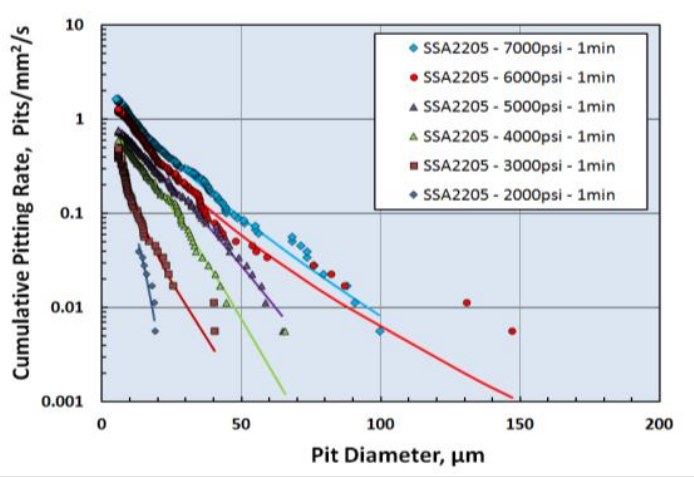

Figure 9: Cumulative pitting rate as a function of pit diameter for different values of the jet pressure on duplex stainless steel A2205 (cut-off depth: $0.5 \mu \mathrm{m})$. Curve fits correspond to the threeparameter Weibull distributions,

$$
N=N^{*} \exp \left(-\left(D / D^{*}\right)^{k}\right) \text {, with all three }
$$
parameters fitted.

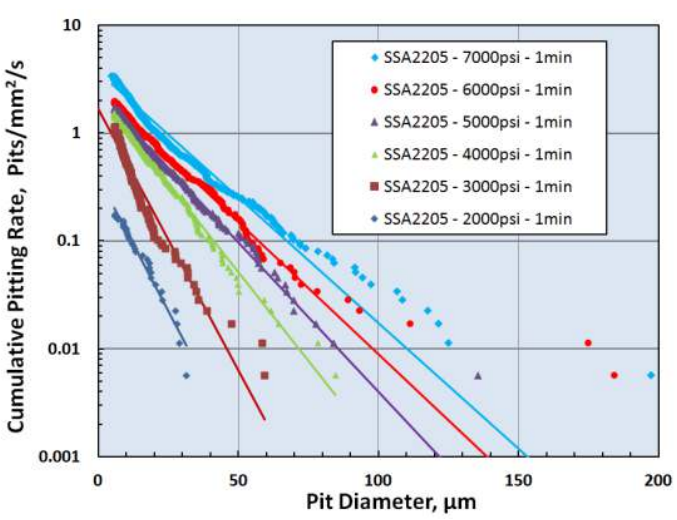

Figure 10: Cumulative pitting rate as a function of pit diameter for different values of the jet pressure on duplex stainless steel A2205 (cut-off depth: $0.3 \mu \mathrm{m})$. Curve fits correspond to Weibull distributions, $N=N^{*} \exp \left[-\left(D / D^{*}\right)^{k}\right]$, with two

\section{parameters fitted and $k=1$.}

Figure 12 and Figure 13 show the two characteristic parameters the pit diameter, $D^{*}$, and the characteristic pitting rate, $N^{*}$, as functions of the jet pressure. One can easily see that the characteristic pit size increases with the jet pressure or speed. This trend can be fitted with a power law indicating that the diameter increases as the jet pressure to the power 0.877 (or the jet velocity to the power 1.75). Similarly, the characteristic pitting rate increases as the jet pressure to the power 1.25 (or the jet velocity to the power 2.5). This high power can be explained by the fact that increasing the jet pressure or speed both brings in more collapsing bubbles and increases the number of more intense cavitation bubble collapse events. Hence, the characteristics pit size and the pitting rate obtained can be used as a measure of the intensity level of the cavitating field.

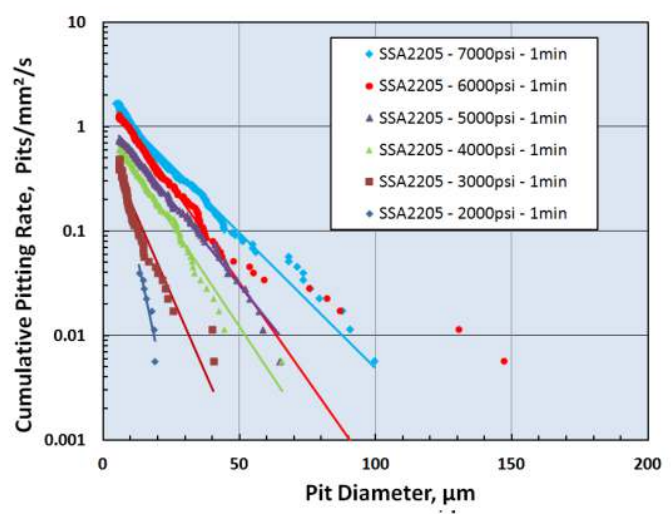

Figure 11: Cumulative pitting rate as a function of pit diameter for different values of the jet pressure on duplex stainless steel A2205 (cut-off depth: $0.5 \mu \mathrm{m})$. Curve fits correspond to parameter Weibull distributions, $N=N^{*} \exp \left[-\left(D / D^{*}\right)^{k}\right]$, with two parameters fitted and $k=1$.

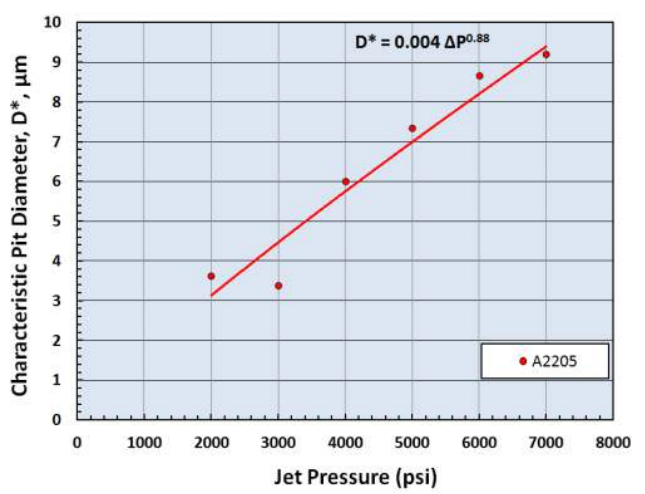

Figure 12. Fitted characteristic parameter, $D *$ for different values of jet pressure on duplex stainless steel A2205 (cut-off depth: $0.3 \mu \mathrm{m}$ ). Here, $k$ is fixed at 0.70 .

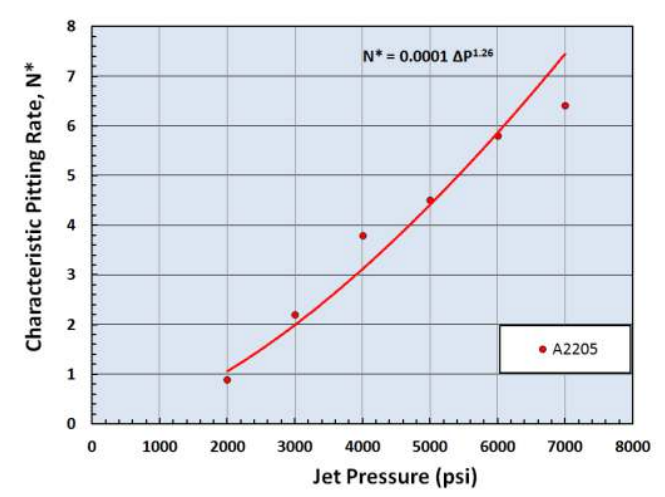

Figure 13. Fitted characteristic parameter, $N *$ for different values of jet pressure on duplex stainless steel A2205 (cut-off depth: $0.3 \mu \mathrm{m}$ ). Here is $k$ is fixed at 0.70 . 


\section{Dependence on Materials}

This section considers the differences between the pitting results between the three considered materials: Aluminum alloy, Nickel Aluminum Bronze, and Duplex Stainless Steel. Figure 14 to Figure 16 show both the cumulative pitting rates as a function of pit diameters for different values of jet pressure and the corresponding Weibull fits with $k=0.7$. For all cases, the cut-off depth was chosen to be $0.30 \mu \mathrm{m}$. One can see that the value of $k=0.7$ can be considered appropriate for all three materials. Figure 16 includes also data for the ultrasonic cavitation method following the G32 standard. The pitting results from two different durations of G32 tests (1 min. vs. $3 \mathrm{~min}$.) produced very close pitting rates. This means during this short time, pit overlapping did not happen. The pitting statistics from G32 tests were close to that of 1000 psi jet cavitation. The G32 data also follows the same functional fit trend.

Using the idea that pitting rates of a specific material in a given erosive cavitation field can be described by the two parameters $\mathrm{N}^{*}$ and $\mathrm{D}^{*}$, we can look at the evolution of these two parameters with both the intensity of the erosive field and the change in material. Figure 17 shows the evolution of $\mathrm{N}^{*}$, the characteristic pitting rate, with the jet pressure for the three materials. We can infer from Figure 18 that the characteristic pit size of NAB is larger compared to that of A2205 for the same erosive filed of load pressures, indicating that A2205 is more resistant to plastic deformation, within the test duration used in this study. This is correlated well with the yield stress values of the two materials as shown in Table 1 . These initial plastic deformations are not necessarily directly related to the cavitation erosion resistance of the materials.

Similarly Figure 18 shows the variations of the characteristic pit size with both the intensity of the erosive field and the change in material. Here too, it is clear that the pit size increases with the jet pressure increases. The value of $D^{*}$ increases almost linearly mildly with the jet pressure (between $\Delta \mathrm{P}^{0.5}$ and $\Delta \mathrm{P}^{1.5}$ ), while the values of $\mathrm{N}^{*}$ increase much more drastically with $\Delta P$, i.e. up to $\Delta \mathrm{P}^{4.5}$ depending on materials. This again highlights that the higher velocity jet cavitation field generates much larger cavitation pressure pulses on the material surface thus making a much larger number of these pulses active in generating pits. On the other hand it seems as if the amplitude of the highest pulses increases in a more moderate way with the jet pressure making the size of the pits increase only almost linearly with the pressure.

\section{Normalization of Results}

The fact that all cumulative pitting rate data seems to follow the same general trends can be clearly illustrated by representing on the same plot all data from all tests after normalizing each cumulative pitting rate data with its corresponding characteristic pitting rate, $\mathrm{N}^{*}$, and after normalizing each pit diameter with the characteristic pit diameters, D*, corresponding to the combination material/jet pressure. As shown in Figure 19, all non-dimensional data is very clearly fitted with the selected Weibull function, $N=N * e^{-\left(\frac{D}{D^{*}}\right)^{k}}$, independent of the considered material and the jet operating conditions. The figure shows two selected values of $k$ : $k=1.0$, which was used in previous work [33] and fits well small diameter pits but not the larger diameter pits, and $k=0.7$, which was found here to be more appropriate over the full range of diameters.

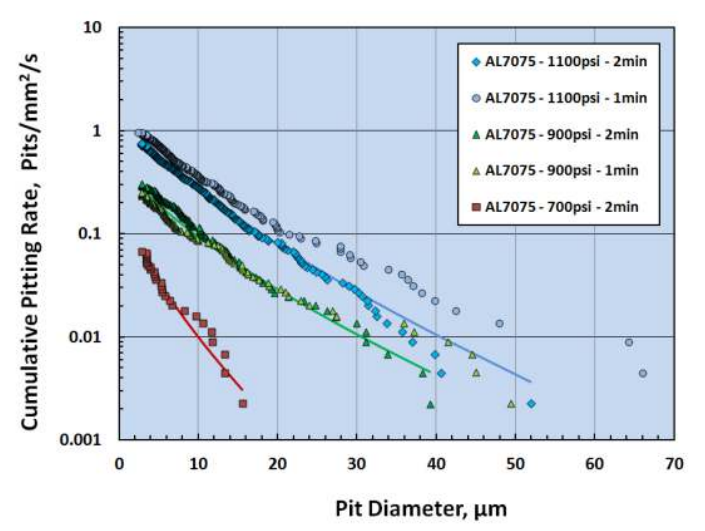

Figure 14: Cumulative pitting rate as a function of pit diameter for different values of the jet pressure on Al7075 (cut-off depth: $0.3 \mu \mathrm{m}$ ). Lines correspond to fitted Weibull distributions with $k=0.70$.

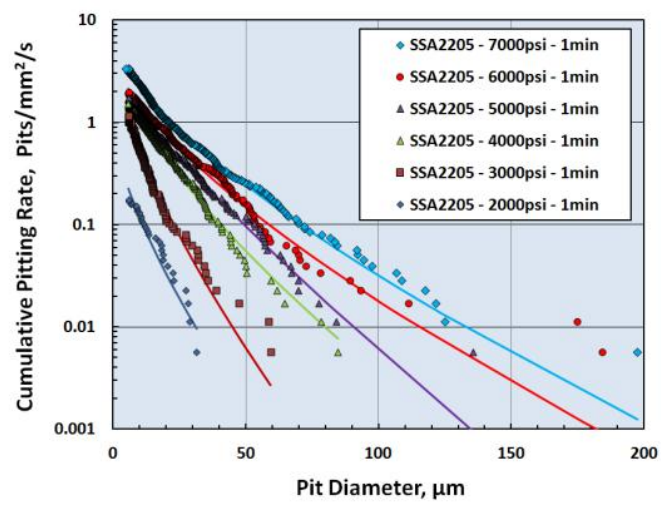

Figure 15: Cumulative pitting rate as a function of pit diameter for different values of the jet pressure on duplex stainless steel A2205 (cut-off depth: $0.3 \mu \mathrm{m}$ ). Lines correspond to fitted Weibull distributions with $k=0.70$. 


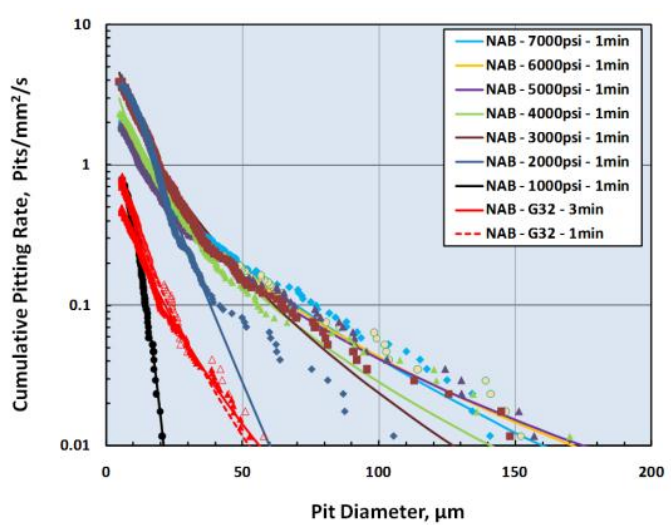

Figure 16: Cumulative pitting rate as a function of pit diameter for different values of the jet pressure on NAB (cut-off depth: $0.3 \mu \mathrm{m}$ ). Lines correspond to fitted Weibull distributions with $k=0.70$.

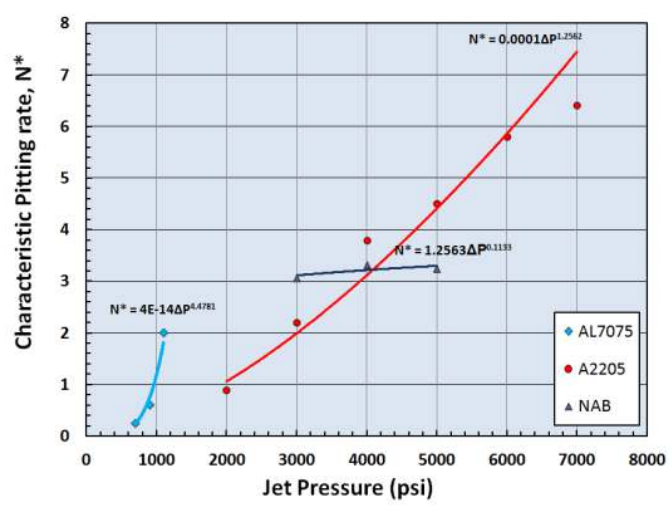

Figure 17. Comparison of fitted characteristic parameter, $N *$ for different values of jet pressure.

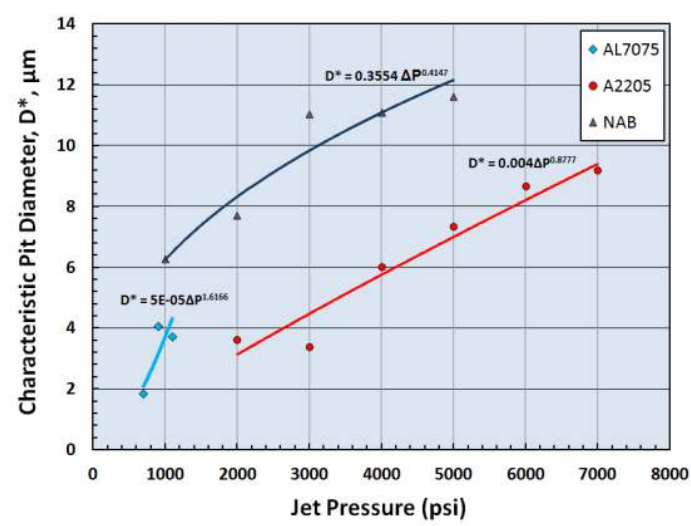

Figure 18. Comparison of the pits characteristic diameter, $D *$, for different values of the cavitation erosive jet pressure.
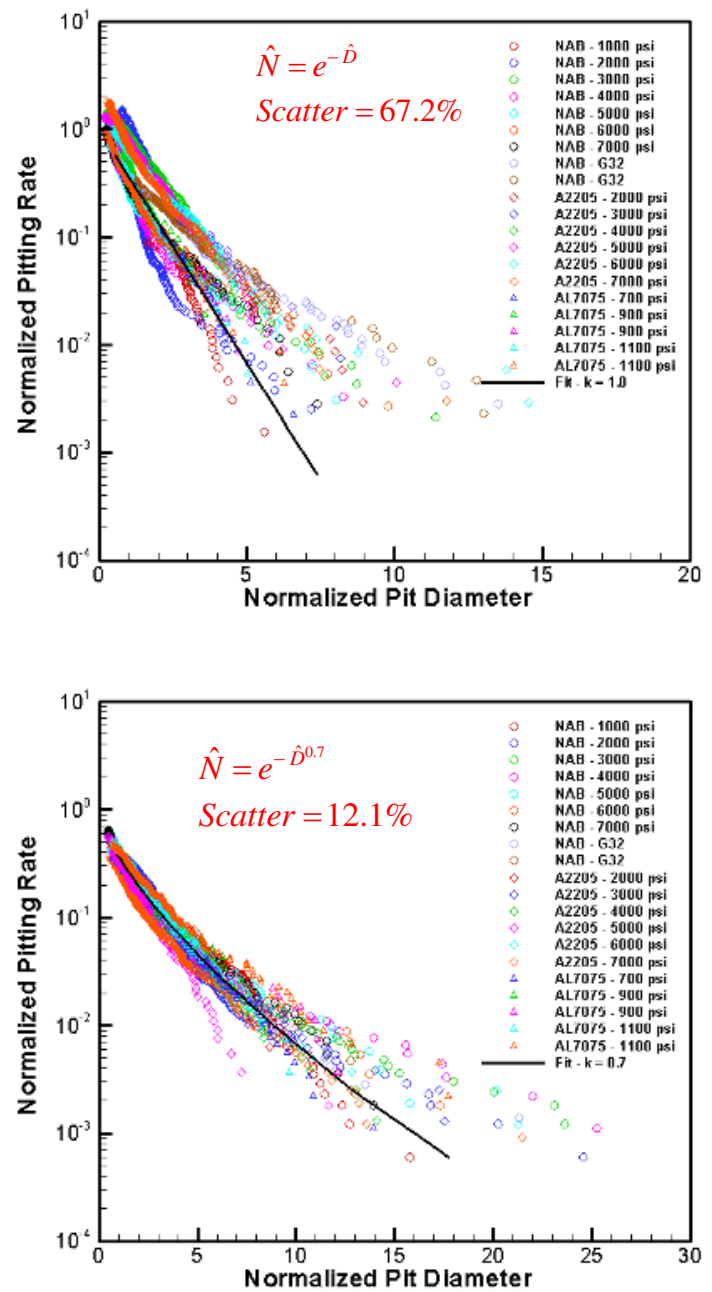

Figure 19. Normalized pitting rate, $N / N^{*}$, vs. normalized pit diameter, $D / D^{*}$, for two values of the fitting function shape factor $k=1.0$ (top) and $k=0.7$ (bottom).

The coverage rate $\beta$, i.e. the fraction of surface covered by all pits whose diameter is larger than a given value $D$, can be computed using Eq. 1 :

$$
\begin{aligned}
\beta(D) & =\int_{D}^{\infty}\left[-\frac{d N}{d D}\right] \frac{\pi D^{2}}{4} d D \\
& =\frac{k \pi}{4} N^{*} D^{* 2} \int_{D / D^{*}}^{\infty} u^{k+1} e^{-u^{k}} d u
\end{aligned}
$$

It can be shown that this $\beta(D)$ function presents a maximum for a particular pit diameter close to $3.6 D^{*}$ in the case $k \cong 0.7$. This particular pit diameter can then be considered as that of the pits which contribute most to the coverage of the pitted surface. This particular pit diameter increases from about $13 \mu \mathrm{m}$ (at $2000 \mathrm{psi}$ ) to $33 \mu \mathrm{m}$ (at $7000 \mathrm{psi}$ ) in the case of stainless steel A2205 as deduced from Fig. 20. These values are somewhat smaller than those given in [33]. This is most likely 
due to an overall smaller length scale of the flow, even though both flows are not geometrically similar.

If all pits are included in the analysis, i.e. if the integral in Eq. 3 is computed from $D / D^{*}=0$ to infinity, the total coverage rate is (still in the case $k \cong 0.7$ ):

$\beta(0) \cong 7.18 \frac{k \pi}{4} N * D *^{2} \cong 4 N * D *^{2}$

The inverse of this total coverage rate, $\tau=1 / \beta(0)$, has the unit of a time and can be interpreted as the coverage time, i.e. the time necessary for the surface to be covered just one time by the pits. This typical time varies between about 2.7 hours (at $2000 \mathrm{psi}$ ) and $8 \mathrm{~min}$ (at $7000 \mathrm{psi}$ ) in the case of stainless steel A2205. This time is one of the most relevant characteristic times of the erosion process since several coverages will actually be needed before complete hardening of the material surface and the subsequent inception of mass loss. Even though the required number of coverages depends upon the level of cavitation in terms of amplitude of impact loads, most characteristic times of the erosion process (such as the incubation time) are expected to be strongly correlated to this coverage time.

\section{Pit Depth and Pit Volume}

The volumes of the pits can be correlated to their equivalent diameter based on the surface area as shown in Figure 20, which presents all individual pits for the duplex stainless steel A2205 samples at the different jet pressures considered. The volumes are calculated based on the full 3D geometry with no assumptions on pit shape, such as axisymmetry or other. The left curve is based on the cutoff depth of $0.3 \mu \mathrm{m}$, while the right curve uses $0.5 \mu \mathrm{m}$. Both figures show that the pit volumes vary like the equivalent diameter raised to the power 2.1, instead of say to the power 3 , which would be expected if the pits were hemispherical. This does not depend significantly upon the depth threshold used and the jet pressures in the present range of investigation. This result is also recovered for all materials tested and for all erosion intensities both with the jets and the ultrasonic cavitation, as illustrated in Figure 21.
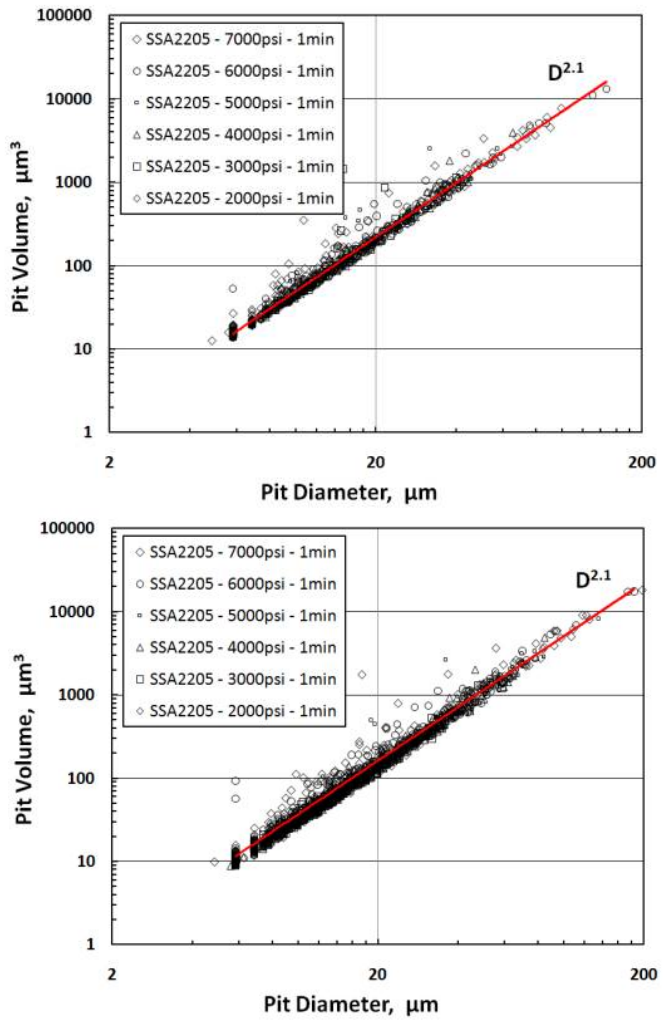

Figure 20: Pit volume as a function of pit diameter for different values of the jet pressure on duplex stainless steel A2205. Left: Cut-off depth: $0.3 \mu \mathrm{m}$,

Righ: cut-off depth: $0.5 \mu \mathrm{m}$. The fit lines correspond to $0.26 D^{2.1}$ (top) and $0.34 D^{2.1}$

(bottom).

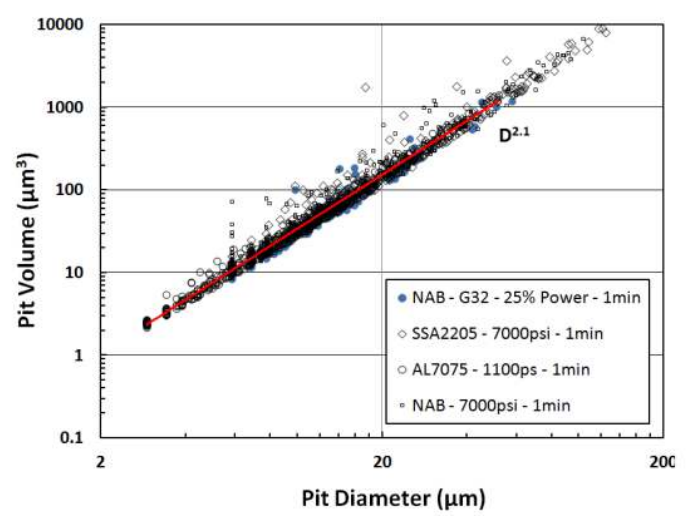

Figure 21: Pit volume as a function of pit diameter for all materials tested and for different cavitation mechanisms.

The small 2.1 exponent, i.e. $v \propto D^{2.1}$ indicates that the plastic deformation is actually much wider than deep, i.e. very shallow. This is illustrated in Figure 22 which shows pit depths over pit diameters, or shape ratio, as a function of pit diameter. The figure also shows the results for the two cutoff depth values: $0.3 \mu \mathrm{m}$ (left) and 0.5 $\mu \mathrm{m}$ (right). The choice of the threshold did not influence the shape factor too much. Figure 22 left 
and Figure 22 right show that a decrease in cut-off depth essentially leads to a translation of the curves towards larger diameters but the different curves remain parallel, which explains why the trend of pit volume with pit diameter is independent of cut-off depth. The shape factor mostly smaller than $10 \%$ but for some rare pits or asperities in the surface, is shown to decrease with increasing diameter approximately as $D^{-0.6}$. Figure 22 also clearly show that there is not a unique pit shape factor but a large variety of mainly shallow pit shapes. An increase in the cutoff depth essentially cuts off more shallow pits.
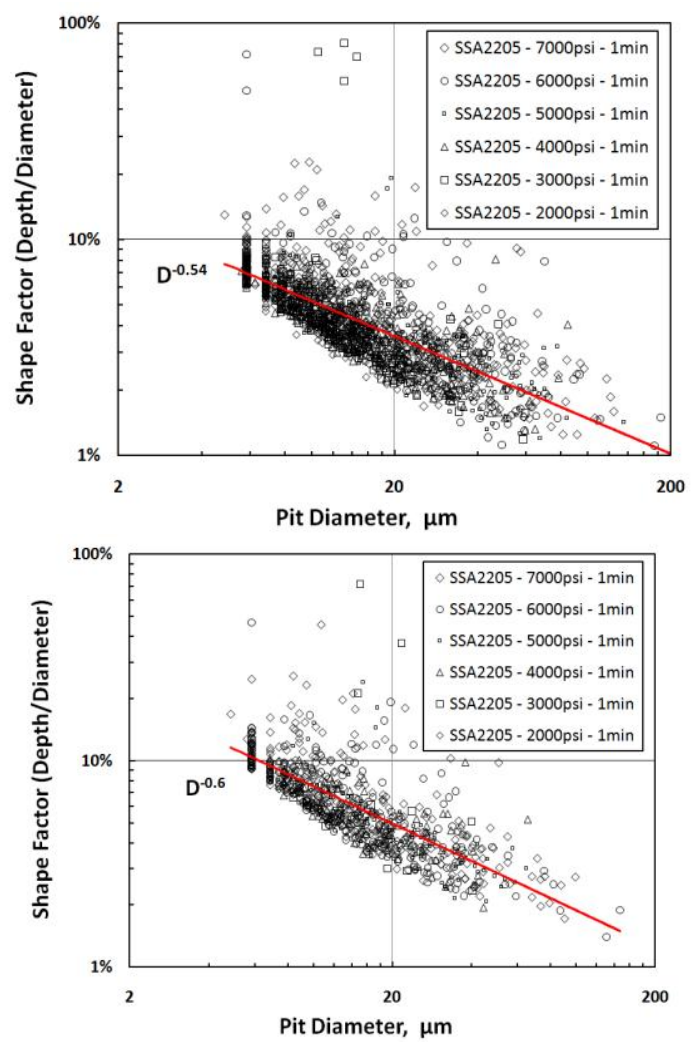

Figure 22: Shape factor or pit depth over pit diameter as a function of pit diameter for different values of the jet pressure on duplex stainless steel 2205. Cut-off depth: 0.3 um (top) vs. cut-off depth: $0.5 \mu \mathrm{m}$ (bottom).

\section{Conclusions}

In order to better characterize cavitation erosion field intensities and the response of metallic materials to these, cavitation erosion pitting tests were conducted on three different materials, an Aluminum alloy, a Nickel Aluminum Bronze, and a Duplex Stainless Steel. The samples were subjected to vastly different intensities of cavitation generated by an acoustic horn and by
DYNAJETS ${ }^{\circledR}$ cavitating jets at different jet pressures. During the short exposure to cavitation field, the sample undergoes permanent plastic deformation, resulting in pit formation. These pits were optically scanned and counted.

Statistical analysis of pitting data revealed that the distribution of pits with diameter can be approximated well by a Weibull three-parameter function, $\quad N=N * e^{-\left(\frac{D}{D^{*}}\right)^{k}}$. One of the parameters is a characteristic pit equivalent diameter, $D^{*}$. Another is a characteristic pitting rate or number of pits per unit area per unit time, $N *$. These two parameters are dependent upon the material and the cavitation intensity. The third parameter in the Weibull curve, the shape factor, $k$, was found to be practically material and cavitation field strength independent. A value of $k=0.7$ was found suitable to capture the overall pit number versus diameter distribution.

The dependence of the erosion field/ material interaction parameters: characteristic diameter, $\mathrm{D}^{*}$, and pitting rate, $\mathrm{N}^{*}$, with the jet pressure, $\Delta \mathrm{P}$, was investigated. For all three materials and all tested pressures, it was found that $D^{*}$ increased almost linearly with $\Delta P$, while the characteristic pitting rate $\mathrm{N}^{*}$ increased more rapidly as say $\sim \Delta \mathrm{P}^{5}$. This illustrates that increasing the jet pressure more intense cavitation activates a much larger number of pressure loads that exceed the material yield stresses and result in pit formation.

The geometric characteristics of the pits were also studied. On average, the pit shapes were similar in terms of pit depth to diameter ratio and pit volume to diameter ratio. These geometric ratios were more or less independent of the materials and the cavitation intensities, and always corresponded to very shallow pits with aspect ratio (depth to diameter) of less than $10 \%$.

\section{Acknowledgement}

Support for this work was provided by Office of Naval Research (ONR) under contract number N00014-08-C-0450, monitored by Dr. KiHan Kim. We would also like to thank many colleagues at Dynaflow for their contributions, comments, and useful discussions; most especially we would like to thank Mr. Patrick Aley, Mr. Emmanuel Coleman, and Mr. Cesar Sanchez for spending many hours using their skills to conduct the tests and making careful measurements and photography. 


\section{Nomenclature}

$\begin{array}{lll}N & \text { Number of Pits } & \# / \mathrm{mm}^{2} / \mathrm{s} \\ N^{*} & \text { Characteristic Number of Pits } & \# / \mathrm{mm}^{2} / \mathrm{s} \\ \tau & \text { Characteristic Time Parameter } & s \\ D & \text { Pit Diameter } & \mu m \\ D^{*} & \text { Characteristic Pit Diameter } & \mu m \\ V & \text { Pit Volume } & \mu m^{3}\end{array}$

\section{References}

1. Thiruvengadam, A., "Handbook of Cavitation Erosion," Hydronautics Technical Report 73011, 1974.

2. Eisenberg, P., Preiser, H.S., and Thiruvengadam, A., "On the Mechanisms of Cavitation Damage and Methods of Protection," Transac. Soc. Naval Architects 73, 241-286, 1965.

3. Pereira, F., Avellan, F., Dupont, Ph., "Prediction of Cavitation Erosion: An Energy Approach," Journal of Fluids Engineering, 120, 719-727, 1998.

4. Choi, J.-K., Chahine, G.L., Frederick, G.S., Aley, P., "Evaluation of Coatings Provided by NSWCCD for Their Resistance to Cavitation Generated by Cavitating DYNAJETS", Report No. 2M7018-NSWC-1, DyNAFLOW, INC. Sep. 2007.

5. Chahine, G.L., "Cavitation Cloud Theory," Proc. 14th Symposium on Naval Hydrodynamics, Ann Arbor, Michigan, National Academy Press, pp. 165-194, Washington, D.C., 1983.

6. Chahine, G. L. and Duraiswami, R., "Dynamical Interactions in a Bubble Cloud", Journal of Fluids Engineering, Vol.114, p.680-686, 1992.

7. Morch, K.A., "Dynamics of Cavitation Bubbles and Cavitating Liquids", Treatise on Materials Science and Technology Vol. 16: 309-355, 1979.

8. Morch, K.A., "Concerted Collapse of Cavities in Ultrasonic Cavitation," Proc. Acoustic Cavitation Meeting, London, 62, 1977.

9. D'Agostino, L. and Brennen, C.E., "On the Acoustical Dynamics of Bubble Clouds", ASME Cavitation and Polyphase Flow Forum, Houston, 72-76, 1983.

10. Brennen, C.E., Cavitation and Bubble Dynamics, Oxford University Press, New York, Oxford, 1995.

11. Shimada, M., Kobayashi, T., Matsumuto, Y., "Dynamics of cloud cavitation and cavitation erosion", Proc. the ASME/JSME Fluids
Engineering Division Summer Meeting, San Francisco, CA, 1999.

12. Blake, J.R., Taib, B.B., Doherty, G., "Transient Cavities near Boundaries. Part I. Rigid Boundary", Journal of Fluid Mechanics, Vol. 170, pp.479-497, 1986.

13. Zhang, Duncan, H., Chahine, G.L., "The Final Stage of the Collapse of a Cavitation Bubble near a Rigid Wall", Journal of Fluid Mechanics, Vol. 257, pp.147-181, 1993.

14. Lauterborn, W., Bolle, H., "Experimental investigations of cavitation-bubble collapse in the neighbourhood of a solid boundary", J. Fluid Mech. 72, 391-399, 1975.

15. Chahine, G.L., Perdue, T.O., "Simulation of the Three-Dimensional Behavior of an Unsteady large Bubble near a Structure", Drops and Bubbles, Third International Colloquium, Monterey, CA, ed. Taylor G. Wang, pp.188199, 1988.

16. Lush, P.A., "Impact of a liquid mass on a perfectly plastic solid", J. Fluid Mech. 135, 373-387, 1983.

17. Parsons, C.A. and Cook, S.S., "Investigation into causes of corrosion or erosion of propellers", J. Am. Soc. Naval Eng., 31, 536541, 1919.

18. Plesset, M.S. and Ellis, "On the mechanism of cavitation damage", A.T., Trans. ASME, 77, 1055-1064, 1955.

19. Knapp, R.T., "Recent Investigations of the Mechanics of Cavitation and Cavitation Damage", Trans. ASME, 77, 1045-1054, 1955.

20. Knapp, R.T., "Accelerated Field Tests of Cavitation Intensity" Trans. ASME, 80, pp.91102, Jan. 1958.

21. Knapp, R.T., Daily, J.W., Hammitt, F.G., Cavitation, McGraw-Hill, London, 1970.

22. Dorey, J.M, Laperrousaz, E., Avellan, P., Dupont, P., Simoneau, R., Bourdon, P., "Cavitation Erosion Prediction on Francis Turbines - Part 3 Methodologies of Prediction", Hydraulic machinery and cavitation: proceedings of the XVIII IAHR Symposium, ed. Cabrera, E., Espert, V. Martinez, F., Vol. I, Kluwer Academic Publishers, 1996.

23. Dular, M., Stoffel, B., Sirok, B., "Development of a cavitation erosion model", Wear 261, 642655, 2006.

24. N. Berchiche, J.P. Franc, J.M. Michel, "A Cavitation Erosion Model for Ductile Materials", Proc. 4th International Symposium on Cavitation, CAV2001, Pasadena, CA, Jun. 2001.

25. Mohamed Farhat, Paul Bourdon, Pierre Lavigne, Raynald Simoneau, "The Hydrodynamic Aggressiveness of Cavitating 
Flows in Hydro Turbines", ASME Fluids Engineering Division Summer Meeting, FEDSM'97, June 1997.

26. Billet, M.L., "The Specialist Committee on Cavitation Erosion on Propellers and Appendages on High Powered/High Speed Ships", Final Report and Recommendations to the 24th ITTC, Proc. the 24th ITTC, Vol. II, 2005.

27. Stinebring, D.R., Holl, J.W, and Arndt, R.E.A., "Two Aspects of Cavitation Damage in the Incubation Zone: Scaling By Energy Considerations and Leading Edge Damage", Journal of Fluid Engineering, Vol. 102, pp. 481485, 1980.

28. Lee, M.K., Hong, S.M., Kim, G.H., Kim, K.H., Rhee, C.K., Kim, W.W., "Numerical correlation of the cavitation bubble collapse load and frequency with the pitting damage of flame quenched Cu-9Al-4.5Ni-4.5Fe alloy", Materials Science \& Engineering A (Structural Materials: Properties, Microstructure and Processing) 425 $(1-2): 15-21$. doi:10.1016/j.msea.2006.03.039, 2006.

29. Hattori S., Takinami, M., Otani, T., "Comparison of cavitation erosion rate with liquid impingement erosion rate", Proc. the 7th International Symposium on Cavitation, Ann Arbor, Michigan, USA, August, 2009.

30. Annual Book of ASTM Standards - Section 3 Material Test Methods and Analytical Procedures, American Society for Testing and Materials (ASTM), Vol. 03.02, 94-109 \& 558571, 2010.

31. Chahine G.L., and Johnson V.E. Jr., "Mechanics and Applications of Self-Resonating Cavitating Jets", International Symposium on Jets and Cavities, ASME, WAM, Miami, FL, Nov. 1985.

32. Choi, J.-K., Jayaprakash, A., Chahine, G.L., "Scaling of Cavitation Erosion Progression with Cavitation Intensity and Cavitation Source", Wear 278-279, 53-61, 2011.

33. Franc, J.-P., Riondet, M., Karimi, A., Chahine, G.L., "Material and Velocity Effects on Cavitation Erosion Pitting", Wear, Wear 274275: pp.248-259. doi:10.1016/j.wear.2011.09.006, 2012.

34. March, P.A., "Evaluating the Relative Resistance of Materials to Cavitation Erosion: a Comparison of Cavitating Jet Results and Vibratory Results", Proc. Cavitation and Multiphase Flow Forum, ASME, Cincinnati, 1987.

35. Dynamic Behavior of Materials, Meyers, M. A., Wiley, p.300, 1994.

36. Carnelli, D., Karimi, A., Franc, J.-P., "Application of Spherical Nanoindentation to Determine the Pressure of Cavitation Impacts from Pitting Tests", Journal of Material Research, 27 (1), 91-99, 2011.

37. "MatWeb Material Property Data", MatWeb LCC, www.matweb.com, 2011.

38. Danzl, R., Helmli, F. S. Scherer, S., "Focus Variation - A New Technology For High Resolution Optical 3D Surface Metrology", 10th Int. Conf. the Slovenian Society for NonDestructive Testing, Ljubljana, Slovenia, September 2009. 